\title{
Punicalagin Induces Serum Low-Density Lipoprotein Influx to Macrophages
}

\author{
Dana Atrahimovich, ${ }^{1,2,3}$ Soliman Khatib, ${ }^{1,2}$ Shifra Sela, ${ }^{3,4}$ \\ Jacob Vaya, ${ }^{1,2}$ and Abraham 0 . Samson ${ }^{3}$ \\ ${ }^{1}$ Department of Oxidative Stress and Human Diseases, MIGAL-Galilee Research Institute, 11016 Kiryat Shmona, Israel \\ ${ }^{2}$ Tel-Hai College, 12208 Upper Galilee, Israel \\ ${ }^{3}$ Faculty of Medicine in the Galilee, Bar-Ilan University, 1311502 Safed, Israel \\ ${ }^{4}$ Eliachar Research Laboratory, Western Galilee Hospital, 22100 Nahariya, Israel
}

Correspondence should be addressed to Abraham O. Samson; avraham.samson@biu.ac.il

Received 24 January 2016; Revised 11 May 2016; Accepted 31 May 2016

Academic Editor: Gabriele Saretzki

Copyright (C) 2016 Dana Atrahimovich et al. This is an open access article distributed under the Creative Commons Attribution License, which permits unrestricted use, distribution, and reproduction in any medium, provided the original work is properly cited.

High levels of circulating low-density lipoprotein (LDL) are a primary initiating event in the development of atherosclerosis. Recently, the antiatherogenic effect of polyphenols has been shown to be exerted via a mechanism unrelated to their antioxidant capacity and to stem from their interaction with specific intracellular or plasma proteins. In this study, we investigated the interaction of the main polyphenol in pomegranate, punicalagin, with apolipoprotein B-100 (ApoB100) that surrounds LDL. Punicalagin bound to ApoB100 at low concentrations $(0.25-4 \mu \mathrm{M})$. Upon binding, it induced LDL influx to macrophages in a concentration-dependent manner, up to 2.5 -fold. In contrast, another polyphenol which binds to ApoB100, glabridin, did not affect LDL influx. We further showed that LDL influx occurs specifically through the LDL receptor, with LDL then accumulating in the cell cytoplasm. Taken together with the findings of Aviram et al., 2000, that pomegranate juice and punicalagin induce plasma LDL removal and inhibit macrophage cholesterol synthesis and accumulation, our results suggest that, upon binding, punicalagin stimulates LDL influx to macrophages, thus reducing circulating cholesterol levels.

\section{Introduction}

Low-density lipoprotein (LDL) particles are the major peripheral tissues providing cholesterol in the human circulation, and they play a key role in the development of atherosclerosis [1]. LDL is surrounded by a single copy of apolipoprotein B-100 (ApoB100) [2] which binds the LDL receptor on the cell surface of target tissues [1].

Punicalagin is a soluble polyphenol isolated from pomegranate with potent antioxidative properties. Punicalagin protects macrophage cells from lipid accumulation and foam cell formation [3-5]. Similarly, coadministration of punicalagin with statin significantly protects against macrophage foam cell formation and inhibits macrophage cholesterol biosynthesis. The use of statins in combination with pomegranate juice in hypercholesterolemic patients enables lowering the dosage of the former, thereby preventing its side effects, such as increases in liver enzymes, muscle problems, cognitive loss, neuropathy, pancreatic and hepatic dysfunction, and sexual dysfunction [6,7]. Pomegranate juice supplementation to atherosclerotic mice reduced macrophage lipid peroxidation, cellular cholesterol accumulation, and development of atherosclerosis [5]. The antiatherogenic effect of punicalagin is known to stem from its antioxidant capacity [8]. However, antioxidant activity cannot be the sole explanation for polyphenols' cellular effects in vivo since they are poorly absorbed through the gut into the bloodstream and extensively metabolized in the small intestine, liver, and colon; thus, their bioavailability is often poor $[9,10]$.

Another antiatherogenic polyphenol is glabridin, isolated from licorice root. Its antiatherogenic properties are assumed to derive from its strong antioxidant capacity [11]. Recently, we showed that, apart from it being an antioxidant, glabridin can protect plasma protein through specific binding [12]. 
In fact, alongside our research, accumulating evidence in the literature suggests that polyphenols interact directly with enzymes, membranes, receptors, and cell or plasma proteins and modulate the activity of key proteins involved in cell signaling [13-16]. Polyphenols' beneficial effect might thus be exerted via a mechanism that is not necessarily related to their antioxidant capacity.

Cells acquire cholesterol through uptake of lipoproteins and through de novo synthesis. Yet (with the exception of steroidogenic tissues), they are unable to catabolize it. Since excess unesterified cholesterol is toxic to cells, organisms have developed several ways to protect themselves from cholesterol accumulation [17]. Macrophages are the best example of this "self-protection"; they take up dead cells containing a large amount of cholesterol, modified lipoproteins, and other extracellular debris. Macrophages take up more cholesterol per cell than any other cell type and protect themselves from cholesterol toxicity by two pathways: one is the esterification of cholesterol to cholesteryl ester. However, accumulation of high levels of cholesteryl ester may lead to the formation of foam cells and, later, to atherogenesis. The second and major line of defense against cholesterol toxicity is high-density lipoprotein (HDL) cholesterol efflux. In addition, in comparison to other cells, macrophages have additional pathways of cholesterol efflux [17]. Excess "peripheral" cholesterol is returned to the liver where the whole-body steady-state level of cholesterol is maintained.

In this study, a possible interaction of punicalagin with ApoB100 and the biological consequences of this interaction were investigated. It was shown that punicalagin binds specifically to ApoB100 and that upon binding it induces LDL influx to macrophages via the LDL receptor; on the other hand, glabridin, which also binds to ApoB100, did not affect LDL influx. These results provide a new mechanism-different from the classical mechanism of "antioxidant activity"-by which punicalagin reduces cholesterol levels in the circulation and attenuates atherosclerosis.

\section{Materials and Methods}

2.1. J774A.1 Macrophage Cell Line. J774A.1 murine macrophage cells were purchased from the American Tissue Culture Collection (ATCC, Rockville, MD). The cells were grown at $37^{\circ} \mathrm{C}, 5 \% \mathrm{CO}_{2}$ in Dulbecco's Modified Eagle's Medium (DMEM) containing glucose (4500 mg/L), $2 \mathrm{mM}$ glutamine, $10 \% \mathrm{w} / \mathrm{v}$ fetal calf serum (FCS), $1 \% \mathrm{w} / \mathrm{v}$ pyruvate, and $0.5 \% \mathrm{w} / \mathrm{v}$ penicillin, streptomycin, and nystatin (all chemicals purchased from Sigma-Aldrich).

2.2. Human LDL Isolation. LDL was prepared from human plasma taken from fasting normolipidemic volunteers (approved for research by Helsinki Committee regulations). It was separated from the plasma by discontinuous density gradient ultracentrifugation [18] and dialyzed against saline with disodium ethylenediaminetetraacetate (EDTA) (1 mM, $\mathrm{pH}$ 7.4). LDL was diluted in phosphate buffered saline (PBS) to $1 \mathrm{mg}$ protein $/ \mathrm{mL}$ and dialyzed twice, for $1 \mathrm{~h}$ each time, and once more overnight against $\mathrm{PBS}$ at $4^{\circ} \mathrm{C}$ to remove EDTA (PBS and EDTA were purchased from Sigma-Aldrich).
2.3. LDL Oxidation. LDL (100 mg protein/L) was incubated with $10 \mu \mathrm{mol} \mathrm{CuSO} / \mathrm{L}$ (Sigma-Aldrich) under gentle shaking for $3 \mathrm{~h}$ at $37^{\circ} \mathrm{C}$. The formation of conjugated dienes was monitored by measuring the increase in absorbance at $234 \mathrm{~nm}$. Measurements were carried out using a SpectraMax M2 Reader [8].

2.4. Fluorescence-Quenching Measurements. Measurements were performed using a previously reported procedure [19]. Briefly, the solution was prepared in a 96-well black enzymelinked immunosorbent assay (ELISA) plate (Greiner BioOne, Germany). To each well, $2 \mu \mathrm{L}$ polyphenol [glabridin, catechin, or quercetin (in ethanol) or punicalagin in double distilled water (DDW)] was added to $5 \mu \mathrm{L}$ LDL or apolipoprotein B-100 (ApoB100) diluted in PBS buffer, to give a final polyphenol concentration in the range of 0.25 to $4 \mu \mathrm{M}$ and a final LDL or ApoB100 concentration of 25 or $10 \mu \mathrm{g}$ protein/mL, respectively. ApoB100 was purchased from Abcam, USA, quercetin and catechin were purchased from SigmaAldrich, glabridin was isolated from licorice root extract [11], and punicalagin was a generous gift from Professor Michael Aviram of the Lipid Research Laboratory, Faculty of Medicine, Technion-Israel Institute of Technology, Haifa.

Fluorescence emission intensity was measured within 30 min of adding the polyphenol to the LDL or ApoB100 solution ( 25 or $37^{\circ} \mathrm{C}$, resp.).

Fluorescence measurements were performed with an Infinite M200 PRO fluorescence spectrophotometer (Tecan) with emission spectra recorded from 320 to $450 \mathrm{~nm}$ at an excitation wavelength of $290 \mathrm{~nm}$. If needed, the inner filter effect, which can decrease the fluorescence intensity, was corrected by using the following relationship:

$$
F_{\text {corr }}=F_{\text {obs }} \times e^{\left(A_{\text {ex }}+A_{\mathrm{em}}\right) / 2}=F_{\text {obs }} \times e^{\left(\varepsilon_{\mathrm{ex}} C L+\varepsilon_{\mathrm{em}} C L\right) / 2},
$$

where $F_{\text {corr }}$ and $F_{\text {obs }}$ are the corrected and observed fluorescence intensities, respectively, $\varepsilon_{\mathrm{ex}}$ is $0.0165 \mu \mathrm{M}^{-1} \mathrm{~cm}^{-1}$ and $\varepsilon_{\mathrm{em}}$ is $0.0034 \mu \mathrm{M}^{-1} \mathrm{~cm}^{-1}$, and $L$ is the well path length $[20,21]$. Fluorescence quenching can occur via two different major mechanisms: static and dynamic. Both quenching pathways are described by the Stern-Volmer equation:

$$
\frac{F_{0}}{F}=1+K_{\mathrm{sv}}[Q]
$$

where $F_{0}$ and $F$ are the fluorescence intensities in the absence and presence of quencher, respectively, $K_{\mathrm{sv}}$ is the Stern-Volmer quenching constant, and [Q] is the quencher concentration. For dynamic quenching, $K_{\mathrm{sv}}$ can be written as $K_{q} \tau_{0}$ :

$$
K_{\mathrm{sv}}=K_{q} \tau_{0}
$$

where $K_{q}$ is the quenching rate constant of the bimolecule and $\tau_{0}$ is the lifetime of the fluorophore in the absence of quencher, which is approximately $10^{-8} \mathrm{~s}$ for a Trp residue [22]. Binding parameters were calculated as described previously [12]. For static quenching, the equilibrium between free and bound molecules can be described by

$$
\log \left(\frac{F_{0}-F}{F}\right)=\log K_{a}+n \log [Q],
$$


where $K_{a}$ is the binding constant, reflecting the degree of interaction between ApoB100/LDL and the polyphenols, and $n$ is the number of binding sites specifying the number of polyphenol molecules bound to the macromolecule. Thermodynamic parameters were calculated as described previously [12]. To characterize the ApoB100-punicalagin interaction, the thermodynamic parameters enthalpy $(\Delta H)$, entropy $(\Delta S)$, and free energy $(\Delta G)$ were calculated. $\Delta H$ can be estimated indirectly by examining the temperature dependence of $K_{a}$ and using (5). $\Delta G$ was estimated from (6) based on the binding constants at different temperatures, and $\Delta S$ was estimated from their relationship (see (7)):

$$
\begin{aligned}
\ln \frac{K_{a 2}}{K_{a 1}} & =\left(\frac{1}{T_{1}}-\frac{1}{T_{2}}\right) \frac{\Delta H}{R}, \\
\Delta G & =-R T \ln K_{a}, \\
\Delta G & =\Delta H-T \Delta S,
\end{aligned}
$$

where $K_{a 1}$ and $K_{a 2}$ are the binding constants at temperatures $T_{1}$ and $T_{2}$, respectively, and $R$ is the gas constant.

2.5. LDL Influx by J774A.1 Macrophages. LDL (1 mg protein $/ \mathrm{mL}$ ) was incubated with $10 \mu \mathrm{g} / \mathrm{mL}$ fluoroisothiocyanate (FITC) (purchased from Pierce, USA) for $1 \mathrm{~h}$ at room temperature in the dark and then dialyzed twice, for $1 \mathrm{~h}$ each time, and once more overnight against carbonate buffer $\mathrm{pH}$ 9) at $4^{\circ} \mathrm{C}$ to remove excess FITC. FITC-conjugated LDL (LDL-FITC) was used for cellular uptake studies. J774A.1 macrophages were incubated at $37^{\circ} \mathrm{C}$ for $3 \mathrm{~h}$ with LDLFITC at a final concentration of $25 \mu \mathrm{g}$ protein $/ \mathrm{mL}$ in DMEM enriched with $20 \%(\mathrm{w} / \mathrm{v}) \mathrm{BSA}$ instead of FCS. LDL uptake was determined by flow cytometry [23]. Measurements of cellular fluorescence were determined by fluorescence-activated cell sorting (FACS) (FACSCalibur 4CA) at $510-540 \mathrm{~nm}$ after excitation at $488 \mathrm{~nm}$ with an argon ion laser. To determine the effect of the polyphenol (glabridin, catechin, quercetin, or punicalagin) on LDL influx, LDL-FITC was incubated with the polyphenol for $15 \mathrm{~min}$ before adding it to DMEM for $3 \mathrm{~h}$.

To confirm that the influx occurs through the LDL receptor, macrophages were incubated simultaneously with LDLFITC $(25 \mu \mathrm{g}$ protein $/ \mathrm{mL})$ and unlabeled LDL $(12,25$, or $50 \mu \mathrm{g}$ protein $/ \mathrm{mL}$ ) to create competitive inhibition.

2.6. Confocal Microscopy Analysis. Macrophages were incubated at $37^{\circ} \mathrm{C}$ for $3 \mathrm{~h}$ with LDL-FITC at a final concentration of $25 \mu \mathrm{g}$ protein $/ \mathrm{mL}$ in the presence or absence of $2 \mu \mathrm{M}$ punicalagin and observed using a Zeiss LSM 700 confocal laser scanning microscope at $63 \mathrm{x}$ magnification. A vertical stack through the $z$-axis of the cells was created with the $488 \mathrm{~nm}$ laser and images were collected at $1 \mu \mathrm{m}$ intervals. Axio Observer.Z1 was used to process the images.

2.7. Statistical Analysis. Statistical analysis was carried out using GraphPad Prism 5.01. Student's paired $t$-test was used to compare the means of two groups. Each experiment was repeated separately at least three times and was always

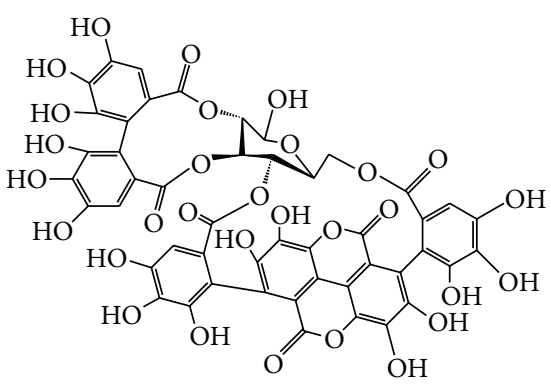

Scheme 1: Punicalagin.

performed in triplicate. Results are presented as mean fluorescence intensity (MFI) with significance determined at $p<0.01(*)$ or $p<0.001(* *)$.

\section{Results}

3.1. Tryptophan- (Trp-) Fluorescence Quenching. Punicalagin (Scheme 1) and glabridin were assayed for possible binding with the LDL particle and its ApoB100 protein. Figure 1 shows the emission spectra of ApoB100 in the presence of various concentrations of punicalagin (Figure 1(a)) and glabridin (Figure 1(b)) and of LDL in the presence of various concentrations of punicalagin (Figure 1(c)) and glabridin (Figure 1(d)) in the $320-415 \mathrm{~nm}$ range with excitation at $290 \mathrm{~nm}$ ( $T=$ $298 \mathrm{~K}, \mathrm{pH} 7$ ). Both glabridin and punicalagin quenched the Trp-fluorescence of ApoB100 and LDL in a concentrationdependent manner. Other polyphenol antioxidants that were examined, such as catechin and quercetin, did not quench the Trp-fluorescence of ApoB100 or LDL (data not shown). The Stern-Volmer curve ( $F_{0} / F$ versus polyphenol concentration), shown in Figure 2, was only linear for the interaction of punicalagin with ApoB100 at the tested concentrations, indicating static or dynamic single-type quenching [24].

Quenching constant $\left(K_{q}\right)$ of ApoB100 initiated by punicalagin was calculated, using (3), to be $3.895 \times 10^{13} \mathrm{M}^{-1} \mathrm{~s}^{-1}$, which is much greater than the maximum diffusion collision quenching rate constant of various drugs with proteins (2 $\left.\times 10^{10} \mathrm{M}^{-1} \mathrm{~s}^{-1}\right)$. This indicated that ApoB100 quenching by punicalagin is not initiated by dynamic collision but via stable complex formation [21, 24]. The ApoB100-glabridin interactions and the interactions with LDL particles, however, were not stable but diffusion dependent and binding parameters of the interaction cannot be determined (Figure 2).

3.2. Binding Constant and Binding Sites. Static quenching was demonstrated for the interaction of ApoB100 with punicalagin by the fact that the Stern-Volmer plot did not show any significant deviation from linearity toward the $y$ - or $x$-axis at the reported punicalagin concentrations (Figure 2) and by the quenching constant $\left(K_{q}\right)$ value (see (3), Section 2). These results suggest a specific interaction between ApoB100 and punicalagin and that the binding parameters can be determined. However, the quenching of ApoB100 and LDL fluorescence by glabridin involves both static and dynamic quenching, as demonstrated by the fact that the Stern-Volmer 


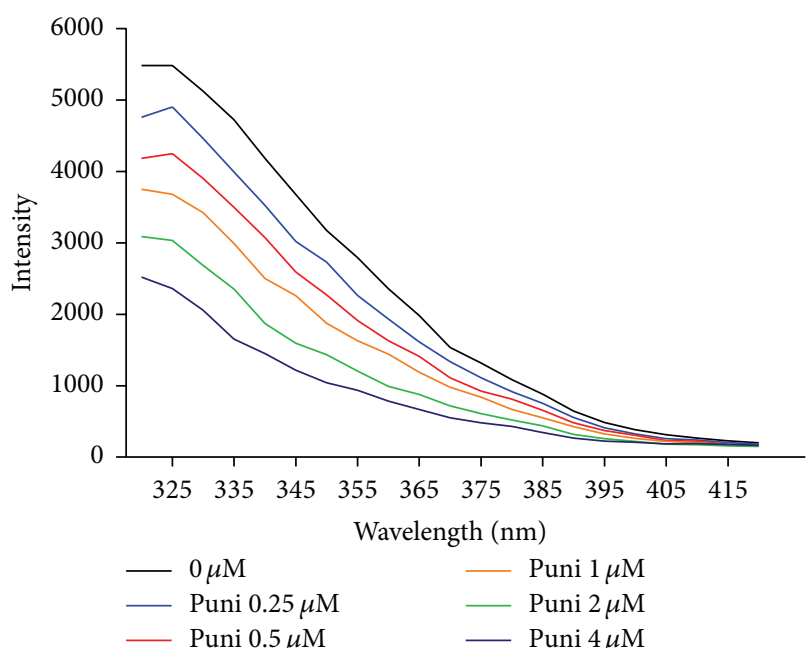

(a)

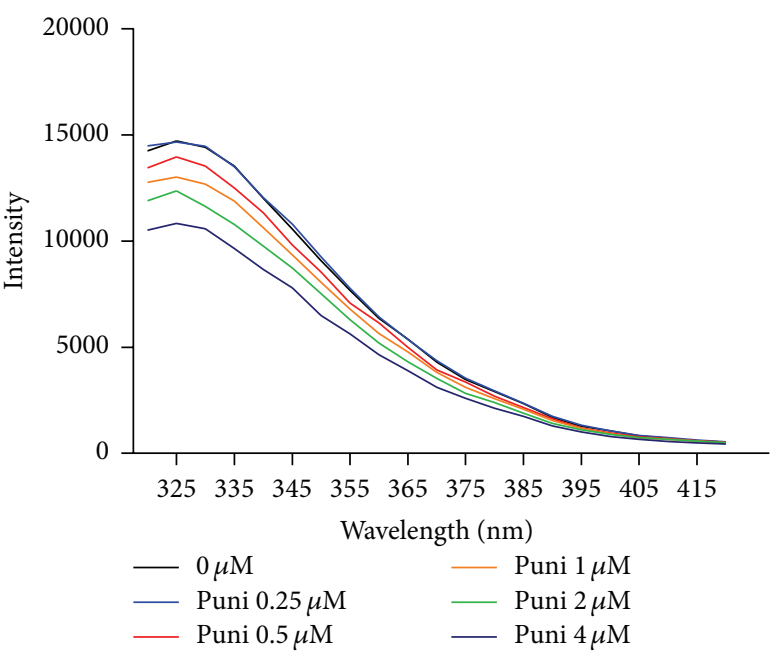

(c)

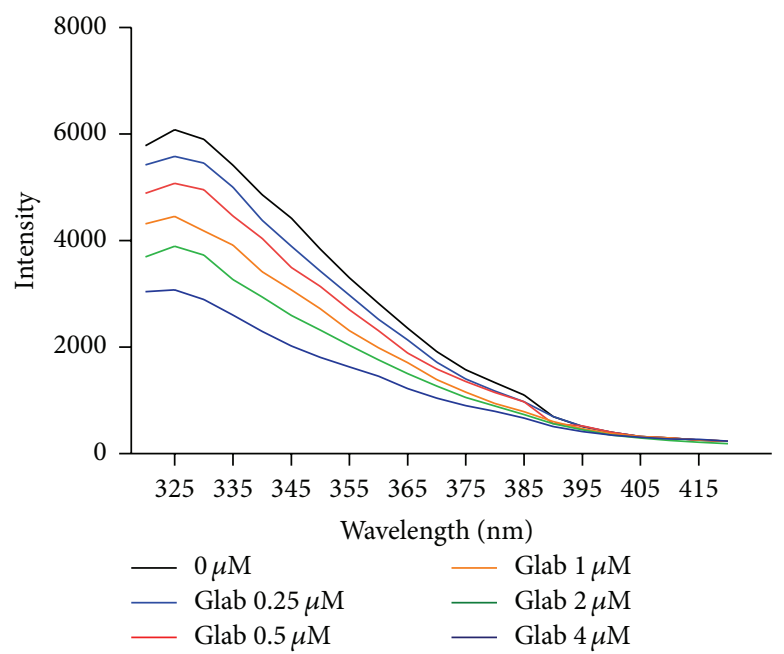

(b)

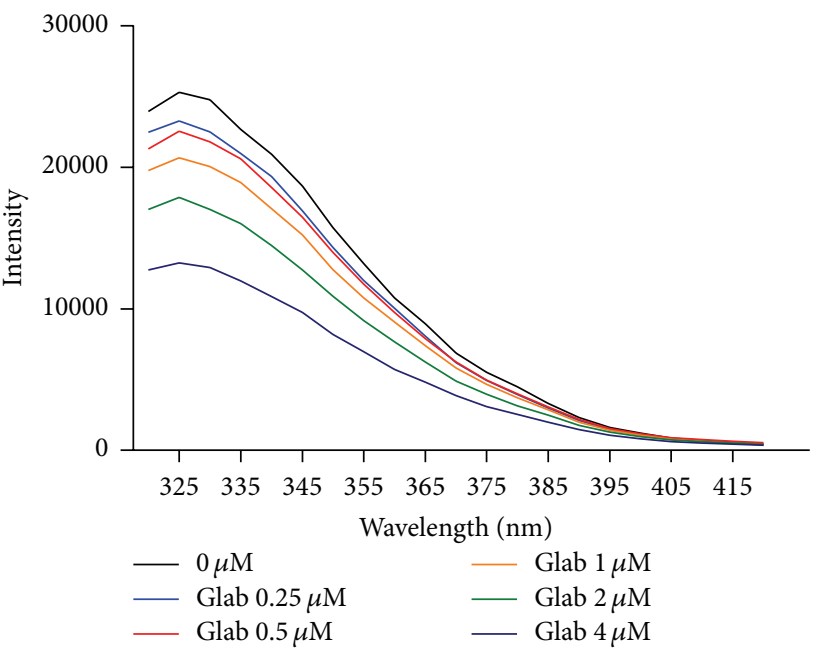

(d)

FIGURE 1: Fluorescence spectra of ApoB100 and LDL in the presence of punicalagin (puni) or glabridin (glab) at various concentrations. Fluorescence spectra of ApoB100 in the presence of various concentrations of punicalagin (a) and glabridin (b) and of LDL in the presence of various concentrations of punicalagin (c) and glabridin (d). In all solutions, the total concentration of ApoB100 and LDL was 0.01 and $0.025 \mathrm{mg} / \mathrm{mL}$, respectively, and the polyphenol concentration was $0.25,0.5,1,2$, and $4 \mu \mathrm{M}\left(\lambda_{\text {ex }}=290 \mathrm{~nm}, \mathrm{pH} 7.4\right.$, and $\left.T=298 \mathrm{~K}\right)$. Each experiment was repeated separately at least three times and was always performed in triplicate.

plot deviates from linearity toward the $x$-axis, which indicates some site inaccessibility [20]. Thus, for glabridin, binding parameters of the interaction cannot be determined.

For static quenching and complex formation the binding parameters between punicalagin and ApoB100 can be determined using (4), Section 2. A plot of $\log \left(F_{0}-F\right) / F$ versus $\log [Q]$, where $Q$ is the polyphenol concentration, was used to determine the binding constant $\left(K_{a}\right), 3.78 \times 10^{6} \mathrm{M}^{-1}$, and the number of binding sites $(n)$ which was close to 1 and not significantly affected by $\mathrm{pH}$ or temperature. These values indicate a single binding site for punicalagin in ApoB100 with a high affinity interaction (Table 1).

3.3. Thermodynamic Parameters and Nature of the Binding Forces. Thermodynamic parameters and nature of the binding forces were calculated for the interaction between punicalagin and ApoB100 using (5), (6), and (7), in Section 2. Table 1 shows negative values for $\Delta G$ and positive values for $\Delta H$ and $\Delta S$. Such thermodynamic results indicate that the interaction is spontaneous and mainly entropy-driven [21].

3.4. LDL Influx to J774A.1 Macrophages. The effect of various polyphenols on LDL influx into macrophages is shown in Figure 3. Figure 3(a) shows that, upon macrophage incubation with $2 \mu \mathrm{M}$ punicalagin, LDL-FITC influx (cell fluorescence intensity) increased from $36 \%$ to $88 \%$. On the other hand, the same concentration of glabridin had no effect on LDL influx (from $36.18 \%$ to $36.09 \%$ ). Interestingly, only punicalagin (in purple) affected LDL influx, as displayed by the curve shift compared to the control curves (in red, black, and brown). No shift was observed for the blue, green, and light-blue curves, representing glabridin, quercetin, and catechin, respectively. 
TABLE 1: Binding constant $\left(K_{a}\right)$, number of binding sites $(n)$, and thermodynamic parameters for the ApoB100-punicalagin interaction (see Scheme 1).

\begin{tabular}{|c|c|c|c|c|c|}
\hline$T(\mathrm{~K})$ & $K_{a}(1 / \mathrm{M})$ & $n$ & $\Delta H(\mathrm{~kJ} / \mathrm{mol})$ & $\Delta G(\mathrm{~kJ} / \mathrm{mol})$ & $\Delta S(\mathrm{~J} / \mathrm{mol} \mathrm{K})$ \\
\hline 310 & $3.78 \cdot 10^{6}$ & $0.95 \pm 0.04$ & \multirow{2}{*}{51.2} & -39.05 & \multirow{2}{*}{291} \\
\hline 298 & $1.7 \cdot 10^{6}$ & $0.7 \pm 0.05$ & & -35.54 & \\
\hline
\end{tabular}

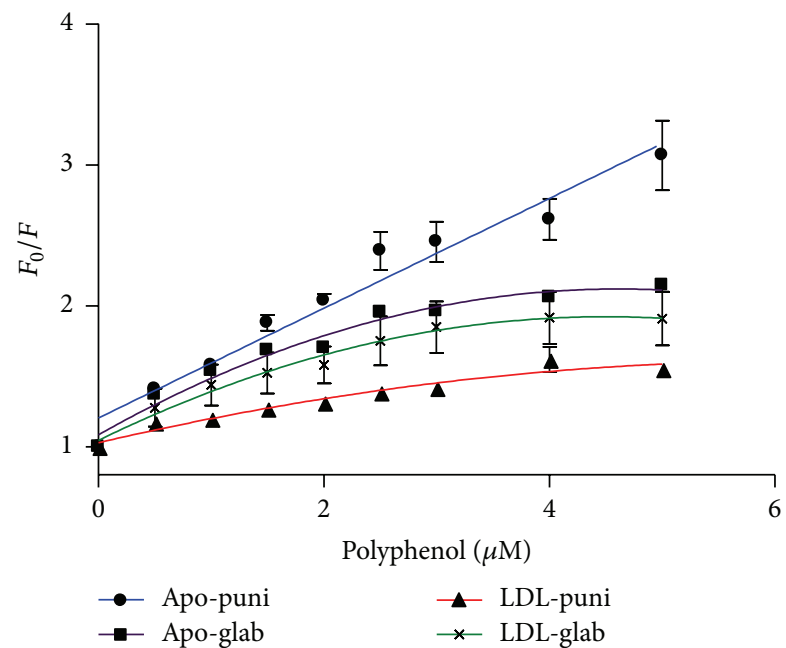

FIGURE 2: Stern-Volmer plots of the fluorescence quenching of ApoB100 and LDL by glabridin (glab) and punicalagin (puni). Each experiment was repeated separately at least three times. Results are presented as mean $\pm \mathrm{SD} . R^{2}=0.9$ and $p<0.0001$ for the linear plot (ApoB100-punicalagin interaction).

Remarkably, LDL influx into the macrophage increased by up to 2.5-fold, mean fluorescence intensity (MFI) from 13 to 35 (Figure 3(b)), only in those cells that were incubated with $2 \mu \mathrm{M}$ punicalagin but not in the cells incubated with glabridin. This finding was unexpected, since both glabridin and punicalagin bind the LDL particle at the same concentrations. It should be noted that, under incubation of oxidized LDL (oxLDL) with macrophages, influx to macrophages is not affected by punicalagin (MFI values were 15.06 and 16.66 in the absence and presence of $2 \mu \mathrm{M}$ punicalagin, resp.). This is because oxLDL penetrates the macrophages through a different receptor, termed "scavenger" receptor (Figure $3(\mathrm{c}))$. The ability of punicalagin to increase LDL influx to macrophages was concentration-dependent. Figure 4(a) demonstrates that while $2 \mu \mathrm{M}$ punicalagin bound to LDL increased cell MFI by $60 \%, 4 \mu \mathrm{M}$ punicalagin increased the LDL influx by $80 \%$.

Next, we attempted to determine whether LDL influx occurs specifically through the LDL receptor. First, the cells were simultaneously incubated with LDL and LDL-FITC in various ratios to create competition. When cells were incubated with LDL $(12 \mu \mathrm{g}$ protein $/ \mathrm{mL})+$ LDL-FITC $(25 \mu \mathrm{g}$ protein $/ \mathrm{mL}$ ), macrophage MFI decreased by $30 \%$; when cells were incubated with an LDL concentration that was twice that of LDL-FITC, MFI decreased by $\approx 45 \%$ (Figure $4(\mathrm{~b})$ ). Finally, images of a vertical $z$ stack of two macrophage cells that were treated with LDL-FITC upon $2 \mu \mathrm{M}$ punicalagin incubation were taken. This image confirms that LDL particles indeed penetrate and accumulate in the cell cytoplasm. In Figure 4(c), a central image of the $z$ stack shows LDLFITC particles accumulated in the cell cytoplasm around the nucleus. It should be noted that, under incubation of oxidized LDL (oxLDL) with macrophages, influx to macrophages is not affected by punicalagin (MFI values were 15.06 and 16.66 in the absence and presence of $2 \mu \mathrm{M}$ punicalagin, resp.). This is because oxLDL penetrates the macrophages through a different receptor, termed "scavenger" receptor.

\section{Discussion}

Dietary polyphenols are found in fruit, vegetables, nuts, and teas [25]. The benefits of consuming polyphenols are commonly assumed to stem from their antioxidant activity, which may contribute to preventing diseases such as cancer, cardiovascular disease, and neurodegenerative disorders [26, 27]. In favor of its antioxidant capacity, punicalagin is antiatherogenic. However, much uncertainty surrounds its mechanism of action as punicalagin concentration in the blood hardly reaches the level needed for effective antioxidant activity (10$100 \mu \mathrm{M})$ [15]. Recent studies suggest that the cellular effects of polyphenols are mediated by their interaction with specific intracellular or plasma proteins [28]. For example, punicalagin interacts with BSA [14]. The present study aimed to investigate a possible interaction of punicalagin with $\mathrm{ApoB} 100$ and the biological consequences of such an interaction.

Trp-fluorescence technique has been widely applied to the study of protein-drug interactions, as changes in the emission spectra of Trp can be seen in response to ligand binding or denaturation [24]. ApoB100 (and LDL) has 37 Trp residues [29] and natural fluorescence quenching can be used to measure its binding affinities. Both glabridin and punicalagin bound LDL or ApoB100 (with no shift in $\lambda_{\mathrm{em}}$ ), while catechin and quercetin did not (Figure 1).

The type of interaction between ApoB100 and punicalagin was interpreted from their fluorescence-quenching spectra [24] and was found to be the only strong and stable one. The values obtained for $n$ indicated a single binding site for punicalagin in ApoB100 (Table 1). Thermodynamic parameters indicated that hydrophobic forces play a major role in the punicalagin-ApoB100 interaction [21].

Macrophages are central to the initiation and progression of atherosclerosis and can be highly appropriate targets for therapy. To examine the biological consequences of polyphenol's interaction with the LDL particle, macrophage cells were incubated with LDL-FITC solution after the latter had been incubated with each polyphenol, to examine the effect of each polyphenol on LDL influx. Similarly, as a negative control, cells were incubated first with the polyphenol without any 

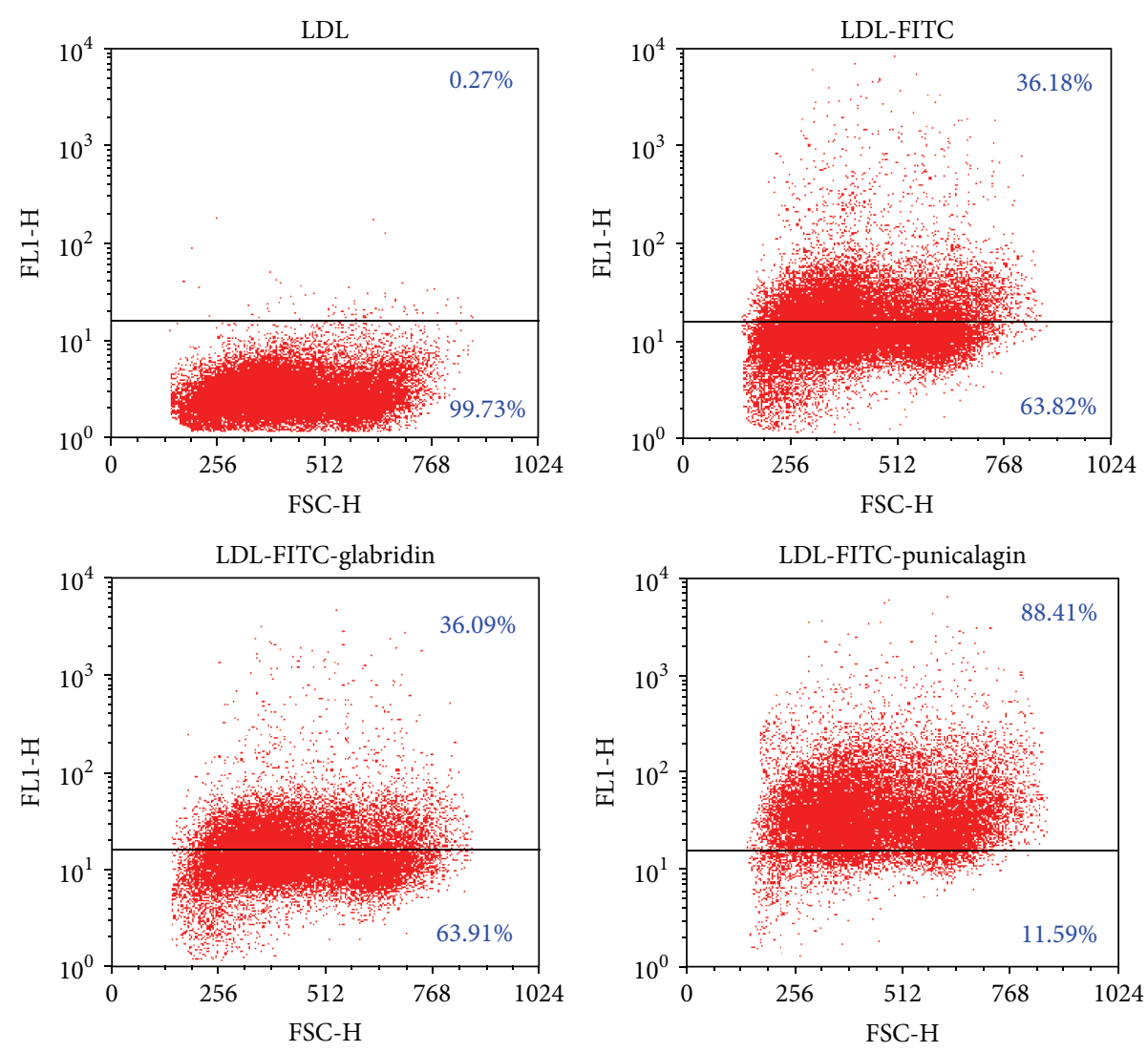

(a)

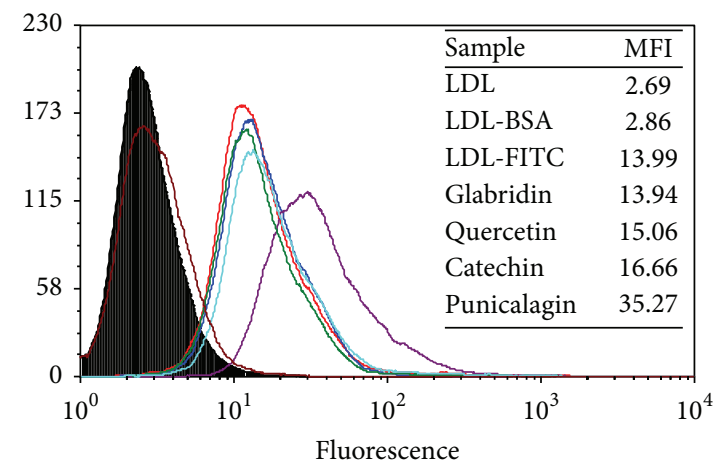

(b)

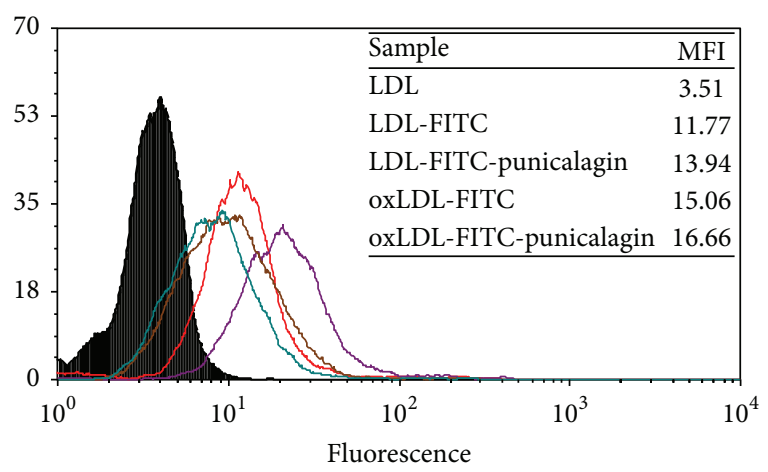

(c)

FIGURE 3: LDL or oxLDL influx into macrophages. (a) The ability of glabridin or punicalagin $(2 \mu \mathrm{M})$ to induce LDL influx to macrophages. (b) FACS histogram of macrophages incubated with LDL-FITC upon addition of $2 \mu \mathrm{M}$ punicalagin (purple), glabridin (blue), quercetin (green), or catechin (light-blue). Note that only punicalagin affects LDL influx as displayed by the curve shift compared to the control curves (red, brown, and black) representing macrophages incubated with LDL-FITC (positive control), LDL (negative control), and FITC-conjugated BSA (negative control), respectively. (c) FACS histogram of macrophages incubated with LDL-FITC (red) upon addition of $2 \mu \mathrm{M}$ punicalagin (purple) or oxLDL-FITC (brown) upon addition of $2 \mu \mathrm{M}$ punicalagin (green). LDL cellular fluorescence was measured in mean fluorescence intensity (MFI) or percent fluorescent cells.

LDL, washed to remove free excess polyphenol, and then incubated with LDL-FITC solution to examine a possible effect of the polyphenol alone on the cell (and in particular on cell LDL receptor). No effects on cells or LDL influx were observed. Supplementary Figure 1 (in Supplementary Material available online at http://dx.doi.org/10.1155/2016/7124251) shows that, upon incubation of the studied cells with
LDL/LDL-FITC for 3 hours, no foam cell formation was evident. The cells did not change their morphology, even after incubation with LDL/LDL-FITC for 16 hours. We may conclude that, under the present experimental conditions, the interaction of punicalagin with LDL leads specifically to LDL influx to the macrophages without their conversion into foam cells. 


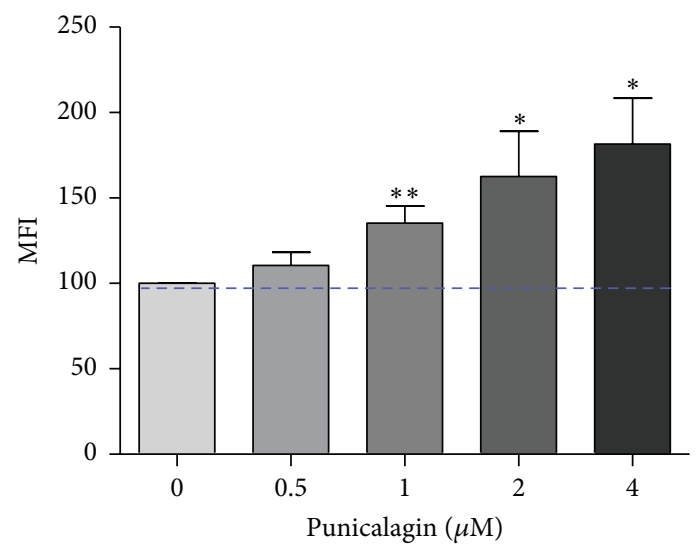

(a)

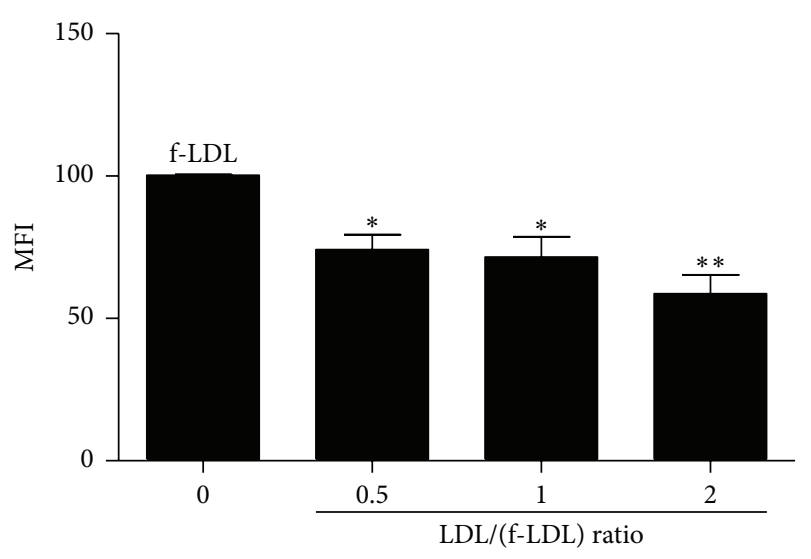

(b)

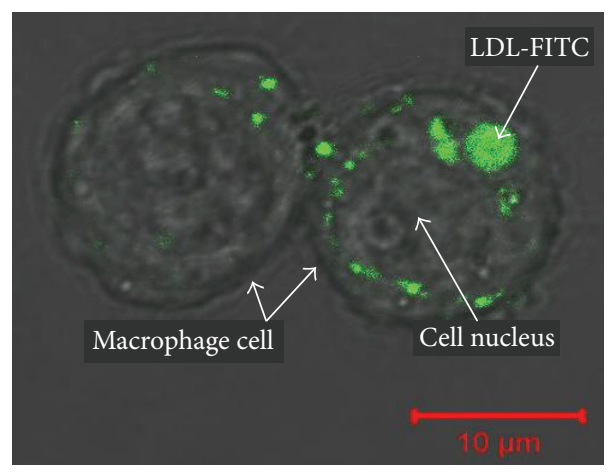

(c)

Figure 4: Punicalagin induces LDL influx. (a) Dose-response effect of 0.5-4 $\mu \mathrm{M}$ punicalagin on LDL influx. (b) Competitive macrophage influx upon adding LDL and LDL-FITC (f-LDL) at various concentrations simultaneously. (c) LDL-FITC particles, upon punicalagin incubation, accumulate in the cell cytoplasm around the nucleus. Each experiment was repeated separately at least three times. LDL cellular fluorescence was measured in MFI with significance determined at $p<0.01(*)$ or $p<0.001(* *)$.

This result highlighted the specificity of the consequences of such interaction between polyphenol and protein (upon macrophages incubation with $2 \mu \mathrm{M}$ punicalagin, LDL influx increased up to 2.5 -fold while the same concentration of glabridin did not affect LDL influx (Figure 3(b))) and that punicalagin induction of LDL influx is in a concentrationdependent manner (Figure 3(c)).

Macrophages are phagocytes that engulf cellular debris and pathogens. We were interested in corroborating the concept that LDL influx occurs specifically through LDL receptor and that macrophages do not take up these particles nonspecifically by endocytosis as part of their defensive activity. FITC reagent was bound to BSA (for which LDL has no known receptor) in the same procedure as LDL-FITC to show that, upon incubation with punicalagin, there is no BSA influx into the cells (Figure 3(b)). In another experiment, competition for LDL receptor was generated by incubating the macrophages with LDL and LDL-FITC simultaneously. Adding LDL in a $1 / 1$ ratio with LDL-FITC led to a $30 \%$ reduction in LDL-FITC influx. Increasing the ratio of LDL to LDL-FITC to $2 / 1$ led to a $45 \%$ reduction in LDL-FITC influx (Figure 4(b)). These results validate the assumption that LDL influx occurs through the LDL receptor.
We postulate that punicalagin binds to ApoB100 in close proximity to the LDL receptor-binding site. Upon binding, punicalagin changes the protein's conformation and might increase LDL's affinity for LDL receptor. Similarly, the conformation of ApoB100 on the surface of the LDL particle is likely to depend on the composition of the core lipids, the surface phospholipid content, and the diameter of the LDL particle [30]. Thus, punicalagin probably interacts with both the lipid part of the LDL particle and the protein, which induces LDL influx into the macrophage. Finally, a vertical $z$ stack of macrophage cells confirmed LDL penetration and accumulation in the cells (Figure 4(c)).

As LDL influx into hepatic cells may contribute to fatty liver disease [31], LDL absorption into hepatic cells in the presence or absence of $2 \mu \mathrm{M}$ punicalagin was also examined (data not shown). Unlike macrophage cells, LDL influx into hepatic cells (hepG2) was not affected by punicalagin when cells were exposed to similar LDL concentrations.

It is important to note that punicalagin from ingestion of pomegranate juice or extract does not reach high concentrations in the blood [15]. It is largely metabolized to ellagic acid through hydrolysis in the small intestine and over time by the gut bacteria to circulating urolithins [32]. 
Therefore, therapeutic administration of punicalagin preferentially should not be oral but rather intravenous. The results presented in this paper are collected from in vitro experiments. We are now examining the in vivo effect of punicalagin, using subcutaneously implanted osmotic minipumps. Serum lipoprotein parameters of mice will be determined after 28 days' exposure to punicalagin.

This study shows that punicalagin binds to a hydrophobic site of ApoB100 and to LDL, which may change the conformation of LDL's bound protein, ApoB100, and enhance its affinity for LDL receptor. LDL influx is induced and cholesterol accumulates in the macrophage cell without foam cell formation. In a future study, the effect of punicalagin on HDL's ability to remove excess cholesterol from these cells to the liver will be explored to determine the mechanism by which punicalagin lowers cholesterol blood concentration as reported in the literature $[4,5,8]$ and attenuates the development of atherosclerosis.

\section{Competing Interests}

The authors declare that they have no competing interests.

\section{References}

[1] M. S. Brown, P. T. Kovanen, and J. L. Goldstein, "Regulation of plasma cholesterol by lipoprotein receptors," Science, vol. 212, no. 4495 , pp. $628-635,1981$.

[2] V. Kumar, S. J. Butcher, K. Öörni et al., "Three-dimensional cryoEM reconstruction of native LDL particles to 16 å resolution at physiological body temperature," PLoS ONE, vol. 6, no. 5, article e18841, 2011.

[3] M. Rosenblat and M. Aviram, "Pomegranate juice protects macrophages from triglyceride accumulation: inhibitory effect on DGAT1 activity and on triglyceride biosynthesis," Annals of Nutrition and Metabolism, vol. 58, no. 1, pp. 1-9, 2011.

[4] M. Aviram, L. Dornfeld, M. Kaplan et al., "Pomegranate juice flavonoids inhibit low-density lipoprotein oxidation and cardiovascular diseases: studies in atherosclerotic mice and in humans," Drugs under Experimental and Clinical Research, vol. 28, no. 2-3, pp. 49-62, 2002.

[5] M. Kaplan, T. Hayek, A. Raz et al., "Pomegranate juice supplementation to atherosclerotic mice reduces macrophage lipid peroxidation, cellular cholesterol accumulation and development of atherosclerosis," Journal of Nutrition, vol. 131, no. 8, pp. 2082-2089, 2001.

[6] M. Rosenblat, N. Volkova, and M. Aviram, "Pomegranate phytosterol ( $\beta$-sitosterol) and polyphenolic antioxidant (punicalagin) addition to statin, significantly protected against macrophage foam cells formation," Atherosclerosis, vol. 226, no. 1, pp. 110-117, 2013.

[7] Z. Reiner, "Resistance and intolerance to statins," Nutrition, Metabolism \& Cardiovascular Diseases, vol. 24, no. 10, pp. 10571066, 2014.

[8] M. Aviram, L. Dornfeld, M. Rosenblat et al., "Pomegranate juice consumption reduces oxidative stress, atherogenic modifications to LDL, and platelet aggregation: studies in humans and in atherosclerotic apolipoprotein E-deficient mice," American Journal of Clinical Nutrition, vol. 71, no. 5, pp. 1062-1076, 2000.
[9] N. P. Seeram, S. M. Henning, Y. Zhang, M. Suchard, Z. Li, and D. Heber, "Pomegranate juice ellagitannin metabolites are present in human plasma and some persist in urine for up to 48 hours," Journal of Nutrition, vol. 136, no. 10, pp. 2481-2485, 2006.

[10] M. Medić-Šarić, V. Rastija, M. Bojić, and Ž. Maleš, "From functional food to medicinal product: systematic approach in analysis of polyphenolics from propolis and wine," Nutrition Journal, vol. 8, article 33, 2009.

[11] B. Fuhrman, S. Buch, J. Vaya et al., "Licorice extract and its major polyphenol glabridin protect low-density lipoprotein against lipid peroxidation: in vitro and ex vivo studies in humans and in atherosclerotic apolipoprotein E-deficient mice," American Journal of Clinical Nutrition, vol. 66, no. 2, pp. 267275, 1997.

[12] D. Atrahimovich, J. Vaya, H. Tavori, and S. Khatib, "Glabridin protects paraoxonase 1 from linoleic acid hydroperoxide inhibition via specific interaction: a fluorescence-quenching study," Journal of Agricultural and Food Chemistry, vol. 60, no. 14, pp. 3679-3685, 2012.

[13] R. J. Williams, J. P. E. Spencer, and C. Rice-Evans, "Flavonoids: antioxidants or signalling molecules?" Free Radical Biology and Medicine, vol. 36, no. 7, pp. 838-849, 2004.

[14] A. P. Kulkarni, H. S. Mahal, S. Kapoor, and S. M. Aradhya, "In vitro studies on the binding, antioxidant, and cytotoxic actions of punicalagin," Journal of Agricultural and Food Chemistry, vol. 55, no. 4, pp. 1491-1500, 2007.

[15] F. Virgili and M. Marino, "Regulation of cellular signals from nutritional molecules: a specific role for phytochemicals, beyond antioxidant activity," Free Radical Biology and Medicine, vol. 45, no. 9, pp. 1205-1216, 2008.

[16] D.-X. Hou and T. Kumamoto, "Flavonoids as protein kinase inhibitors for cancer chemoprevention: direct binding and molecular modeling," Antioxidants and Redox Signaling, vol. 13, no. 5, pp. 691-719, 2010.

[17] M. Cuchel and D. J. Rader, "Macrophage reverse cholesterol transport: key to the regression of atherosclerosis?" Circulation, vol. 113, no. 21, pp. 2548-2555, 2006.

[18] M. Aviram, "Plasma lipoprotein separation by discontinuous density gradient ultracentrifugation in hyperlipoproteinemic patients," Biochemical Medicine, vol. 30, no. 1, pp. 111-118, 1983.

[19] D. Atrahimovich, J. Vaya, and S. Khatib, "The effects and mechanism of flavonoid-rePON1 interactions. Structure-activity relationship study," Bioorganic and Medicinal Chemistry, vol. 21, no. 11, pp. 3348-3355, 2013.

[20] A. Shpigelman, G. Israeli, and Y. D. Livney, “Thermally-induced protein-polyphenol co-assemblies: beta lactoglobulin-based nanocomplexes as protective nanovehicles for EGCG," Food Hydrocolloids, vol. 24, no. 8, pp. 735-743, 2010.

[21] Y. Sun, H. Zhang, Y. Sun et al., "Study of interaction between protein and main active components in Citrus aurantium L. by optical spectroscopy," Journal of Luminescence, vol. 130, no. 2, pp. 270-279, 2010.

[22] L. Liang, H. A. Tajmir-Riahi, and M. Subirade, "Interaction of $\beta$ Lactoglobulin with resveratrol and its biological implications," Biomacromolecules, vol. 9, no. 1, pp. 50-56, 2008.

[23] D. A. Bass, J. W. Parce, and L. R. Dechatelet, "Flow cytometric studies of oxidative product formation by neutrophils: a graded response to membrane stimulation," Journal of Immunology, vol. 130, no. 4, pp. 1910-1917, 1983.

[24] J. R. Lakowicz, Principles of Fluorescence Spectroscopy, Springer, Berlin, Germany, 2006. 
[25] C. Manach, A. Scalbert, C. Morand, C. Rémésy, and L. Jiménez, "Polyphenols: food sources and bioavailability," The American Journal of Clinical Nutrition, vol. 79, no. 5, pp. 727-747, 2004.

[26] M. R. Peluso, "Flavonoids attenuate cardiovascular disease, inhibit phosphodiesterase, and modulate lipid homeostasis in adipose tissue and liver," Experimental Biology and Medicine, vol. 231, no. 8, pp. 1287-1299, 2006.

[27] E. Grotewold, The Science of Flavonoids, 2006.

[28] J. Xiao and G. Kai, "A review of dietary polyphenol-plasma protein interactions: characterization, influence on the bioactivity, and structure-affinity relationship," Critical Reviews in Food Science and Nutrition, vol. 52, no. 1, pp. 85-101, 2012.

[29] S. Bonneau, C. Vever-Bizet, P. Morlière, J.-C. Mazière, and D. Brault, "Equilibrium and kinetic studies of the interactions of a porphyrin with low-density lipoproteins," Biophysical Journal, vol. 83, no. 6, pp. 3470-3481, 2002.

[30] C. Flood, M. Gustafsson, R. E. Pitas, L. Arnaboldi, R. L. Walzem, and J. Borén, "Molecular mechanism for changes in proteoglycan binding on compositional changes of the core and the surface of low-density lipoprotein-containing human apolipoprotein B100," Arteriosclerosis, Thrombosis, and Vascular Bio$\log y$, vol. 24, no. 3, pp. 564-570, 2004.

[31] K. L. Ma, X. Z. Ruan, S. H. Powis, Y. Chen, J. F. Moorhead, and Z. Varghese, "Inflammatory stress exacerbates lipid accumulation in hepatic cells and fatty livers of apolipoprotein E knockout mice," Hepatology, vol. 48, no. 3, pp. 770-781, 2008.

[32] D. Heber, "Pomegranate ellagitanins," in Herbal Medicine: Biomolecular and Clinical Aspects, chapter 10, CRC Press, 2nd edition, 2011. 


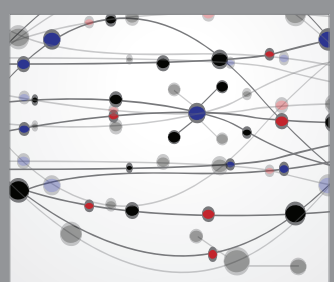

The Scientific World Journal
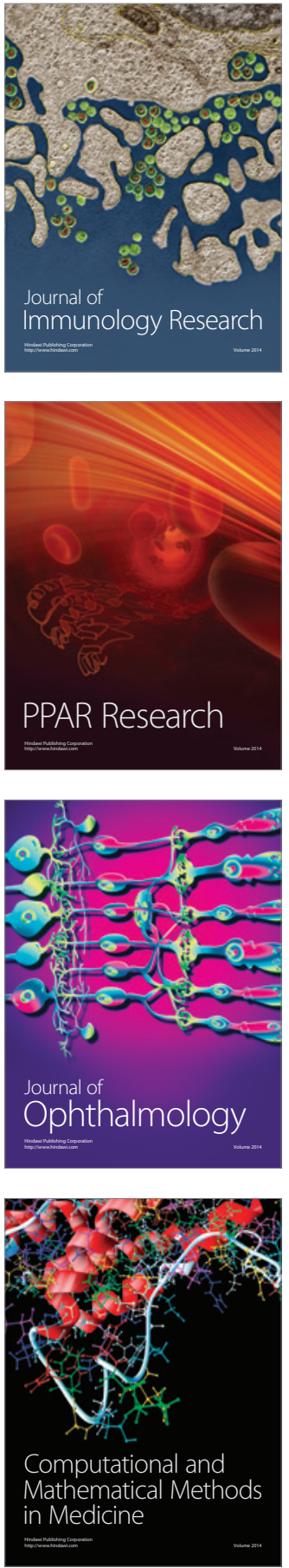

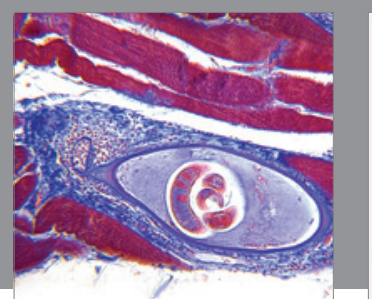

Gastroenterology Research and Practice

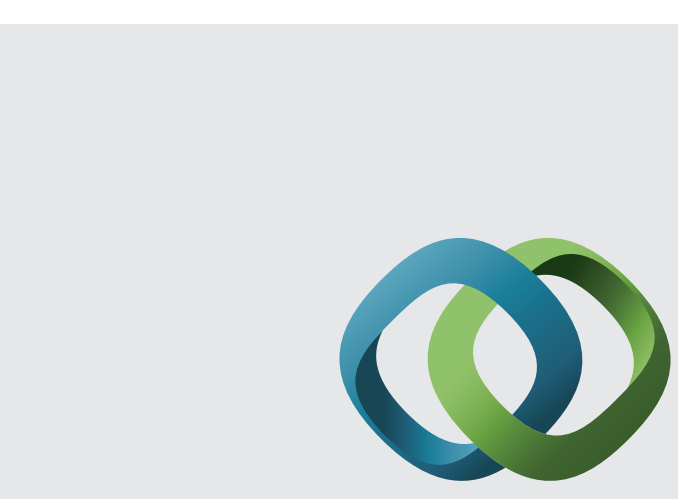

\section{Hindawi}

Submit your manuscripts at

http://www.hindawi.com
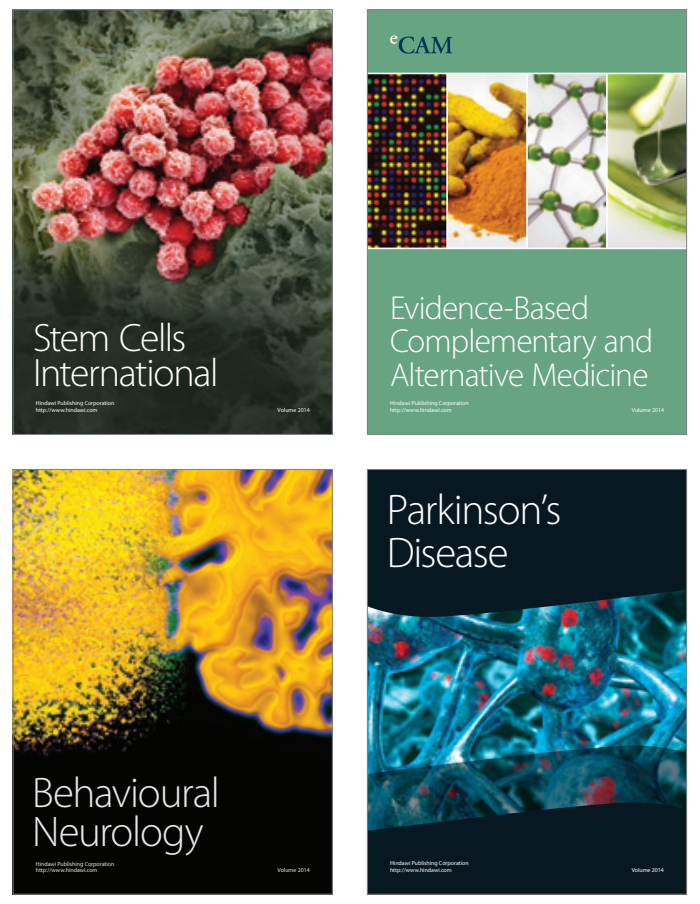
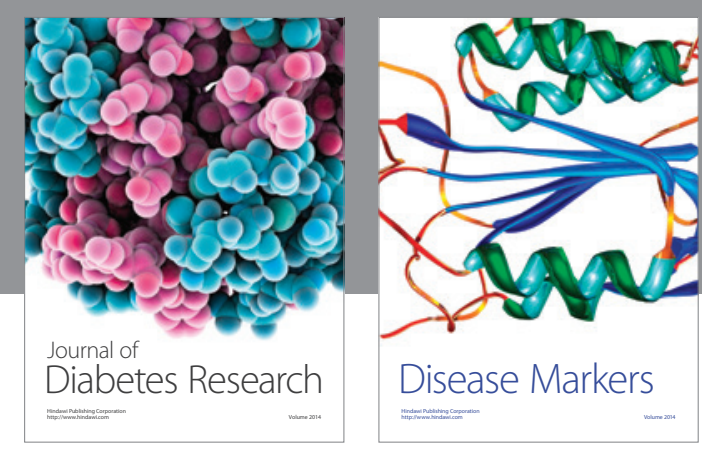

Disease Markers
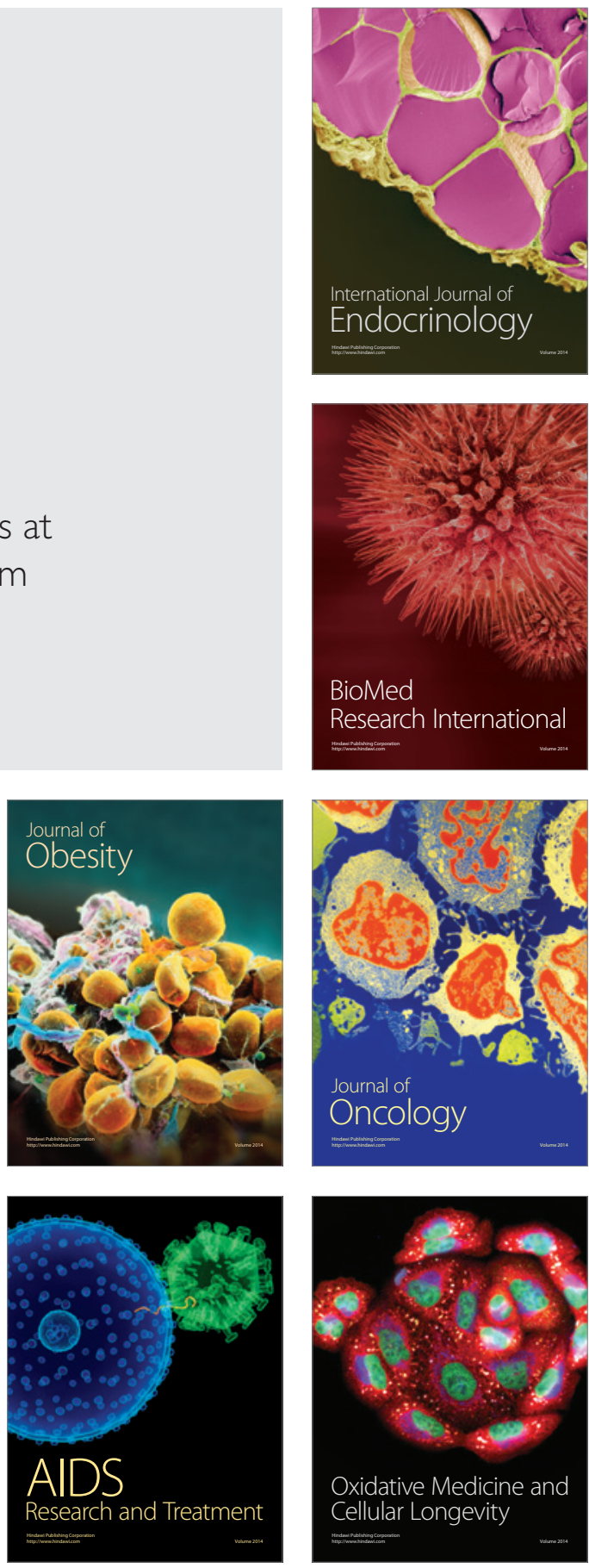